C2008 IEEE. Personal use of this material is permitted. However, permission to reprint/republish this material for advertising or promotional purposes or for creating new collective works for resale or redistribution to servers or lists, or to reuse any copyrighted component of this work in other works must be obtained from the IEEE. 


\title{
Ascertaining the Semantic and Linguistic Level of Perceived Risk in e- Business Interactions
}

\author{
Omar Hussain, Elizabeth Chang, Tharam Dillon and Farookh Hussain \\ Digital Ecosystems and Business Intelligence Institute, Curtin University of Technology \\ o.hussain@cbs.curtin.edu.au
}

\begin{abstract}
By analyzing the level of perceived risk in the domain of e-business, the interaction initiating agent can determine beforehand whether or not it will achieve its desired outcomes and the associated consequences to it in interacting with the other agent. In our previous work, we have proposed a methodology by which the initiating agent ascertains the numeric level of perceived risk in forming an interaction. In this paper, we propose a methodology by which the initiating agent of the interaction determines the semantic level of perceived risk, for it to be utilized while making an informed interactionbased decision with an agent.
\end{abstract}

\section{Introduction}

Business interactions form the backbone which drives the economy of the modern world. Those interactions are carried out with the aim of achieving certain specific outcomes that are consequential for the progression, advancement and sustenance of the particular business or individual. Failure to achieve those specific outcomes might have far reaching consequences to the business or individual. One of such important consequences as a result of failure of such interactions is the experience of financial loss. This level of financial loss that could be experienced can be ascertained by analyzing the level of perceived risk in forming an interaction. Risk highlights the threats and the impact that those threats have on the object at stake in the interaction. These negative aspects in an interaction cannot be determined by determining either the level of trust or security in the interaction. In the literature researchers have defined risk by associating it with an unbiased outcome [1]. But the reality is that an unbiased event might not change the outcome of the interaction that is 'unwanted' in the interacting agent view as much as the negative outcome does, and hence we consider that risk in an interaction is associated with the occurrence of negative outcomes in it. In our previous work, we consider that risk in e-business interactions is a combination of two sub-categories, namely performance risk and financial risk. We term the two agents participating in an interaction as the 'risk assessing agent' and 'risk assessed agent'. The former refers to the one initiating the interaction while the latter refers to the one with whom it interacts with, to achieve its desired outcomes. Performance risk represents the likelihood and the magnitude to which the risk assessing agent will not achieve its expectations in interacting with a risk assessed agent. Financial risk represents the impact on the object at stake in the interaction due to the level of failure in it. Due to space limitations, we will not discuss the approaches to determine these subcategories of risk in this paper. Interested readers are requested to refer to Hussain et al. [2] and Hussain et al. [3] where we have explained in detail the approaches to determine the sub-categories of perceived risk in e-business. In this paper we extend on our previous work of determining the numerical level of perceived risk and propose an approach by which the risk assessing agent determines and quantifies the linguistic level/s of perceived risk in forming an interaction with the risk assessed agent, by utilizing its determined subcategories. We utilize a fuzzy inference system to ascertain semantically the level of perceived risk in an interaction. The proposed methodology is explained in the next sections.

\section{Ascertaining the Semantic level of Perceived Risk in an Interaction}

We consider that the risk assessing agent from the determined subcategories considers the following constituents to determine the linguistic level of perceived risk:

- The Loss of Investment Probability, and

- The Possible Consequences of Failure. 
This is consistent with the approach that we proposed in Hussain et al. [4] where the risk assessing agent, by utilizing these constituents determines the numerical level of perceived risk in forming an interaction. The 'Loss of Investment Probability' (LOIP) in the interaction gives the probability of the risk assessing agent not achieving the full benefit of its resources that it invests while interacting with a risk assessed agent. The 'Possible Consequences of Failure' (PCF) in a business interaction represents the additional degree/s of resources which the risk assessing agent has to keep at stake from its maximum investment capacity, while interacting with the risk assessed agent. Our motive for incorporating the fuzzy inference system is for the risk assessing agent to utilize the fuzzy sets and rules to combine the different determined constituents and ascertain semantically the level of perceived risk in interacting with a risk assessed agent. So an overview of our proposed fuzzy inference model with the variables which it takes as inputs and based on that computes an output level of perceived risk can be classified as:

Inputs:

- Possible Consequences of Failure to the risk assessing agent in interacting with the risk assessed agent (PCF);

- Loss in Investment Probability to the risk assessing agent in interacting with the risk assessed agent (LOIP).

Output:

- Level of Perceived Risk in the interaction (PR).

Fuzzy inference systems are mathematical objects modeling the vagueness present in the natural language when the described phenomena do not have sharply defined boundaries. As mentioned in the literature, fuzzy systems were developed to incorporate the concept of partial truth characterized by the fuzziness of the data which yields a more accurate mathematical representation of the perception of truth than that of crisp sets [5]. A fuzzy inference system models the vague inputs in terms of semantics and transforms them into a mathematical representation of the data to map its output semantics. To achieve this, fuzzy inference system transforms each input from crisp data to fuzzy sets. In order to do that, the fuzzy inference system needs to first have respective data from each input variable to transform it into fuzzy sets. In order to determine the distribution of each input constituents (PCF and LOIP), we define the scope or the universe of discourse (UoD) within which each of the particular variable exists by the following sets:

Possible Consequences of Failure $(P C F)=\{0,1,2$, $3 \ldots \ldots . . .100\}$ where each element has a unit of $\%$.
Loss of Investment Probability (LOIP) $=\{0,1,2$, $3 \ldots \ldots \ldots \ldots . .100\}$ where each element has a unit of $\%$. Perceived Risk in an Interaction $(P R)=\{0,1,2$, 3 ............100 $\}$ where each element has a unit of $\%$.

In the next sub-section we will define the membership functions of the inputs and the output to the fuzzy inference system.

\subsection{Defining the Fuzzy sets and the Membership function for the Input: Possible Consequences of Failure (PCF)}

To classify different fuzzy sets for the input variable 'Possible Consequences of Failure', we divide the universe of discourse such that there are 6 predicates in it. The predicates defined for the input variable are: 'Extremely Low', 'Low' 'Low Medium', 'Medium High', 'High' and 'Extremely High'. The membership function of the linguistic variable 'Possible Consequences of Failure' is represented by the trapezoidal curve such that its shape is as shown in Figure 1.

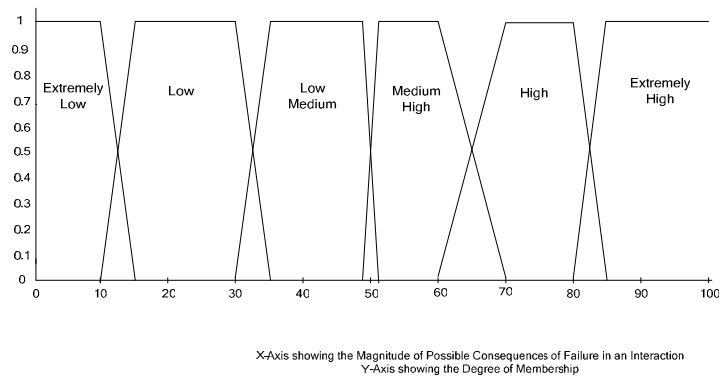

Figure 1: Membership function of the input: Possible Consequences of Failure

\subsection{Defining the Fuzzy sets and the Membership function for the Input: Loss of Investment Probability (LOIP)}

The input given by the risk assessing agent to the fuzzy inference system for the linguistic variable 'Loss of Investment Probability' is a crisp value within the range of 0-100 [3]. To transform the crisp value into a fuzzy value, we define six different fuzzy sets namely 'Extremely Low', 'Low', 'Low Medium', 'Medium High', 'High' and 'Extra High'. Within these different predicates, the degree of truth to which the input value of LOIP quantifies is determined by the membership function. We define the membership function of the linguistic variable 'Loss of Investment Probability' in Figure 2. 


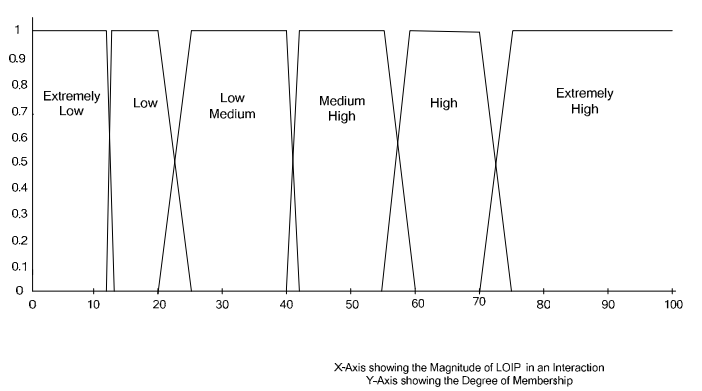

Figure 2: Membership function of the input: Loss of Investment Probability

The focal elements of each input variable are determined by the possibility of occurrence or each element from their UoD as mentioned in Hussain et al. [4]. To transform a focal element ' $x$ ' of an input linguistic variable to the defined fuzzy sets in it, the risk assessing agent has to determine the possibility to which that element ' $x$ ' corresponds with the defined predicates of that input variable, by considering the overlap between the degree of evidence of the input value ' $x$ ', with the degree of membership to which that input value ' $x$ ' corresponds to a particular predicate from the membership function for that input variable. The possibility that the fuzzy set or predicate ' $A$ ' of an input linguistic variable will occur based on the degree of evidence of input ' $x$ ' is given by [5]:

$$
\Pi(\mathrm{A})=\max \left\{\min \left[\pi(\mathrm{x}), \mathrm{DOM}_{\mathrm{A}}(\mathrm{x})\right]\right\} \quad \text { Equation } 1
$$

Equation 1 is repeated for each focal element ' $\mathrm{x}$ ' from the UoD for an input variable to determine the possibility of occurrence of a fuzzy set or predicate 'A'. Once all the input variables have been transformed to their corresponding fuzzy sets, they must then be processed in the inference engine of the fuzzy system to draw a conclusion on the UoD of the output linguistic variable, based on the given evidences on the fuzzy variables that it computes from the inputs. In the next sub-section, we define the output linguistic variable and propose its membership functions.

\subsection{Defining the Fuzzy set and the Membership function for the Output: Perceived Risk (PR) in the Interaction}

The fuzzy inference system based on the inputs given to it computes an output specifying the magnitude and level of perceived risk present in the interaction. The universe of discourse (UoD) of the output membership function 'Perceived Risk' is in the range of $\{0,1,2,3$, $4,5 \ldots \ldots 100\}$. We divide the UoD into six different fuzzy sets by using the predicates, 'Extremely Low',
'Low', 'Low Medium', 'Medium High', 'High' and 'Extremely High'. We define the membership function of the output 'Perceived Risk' in an interaction by using a trapezoidal curve as shown in Figure 3.
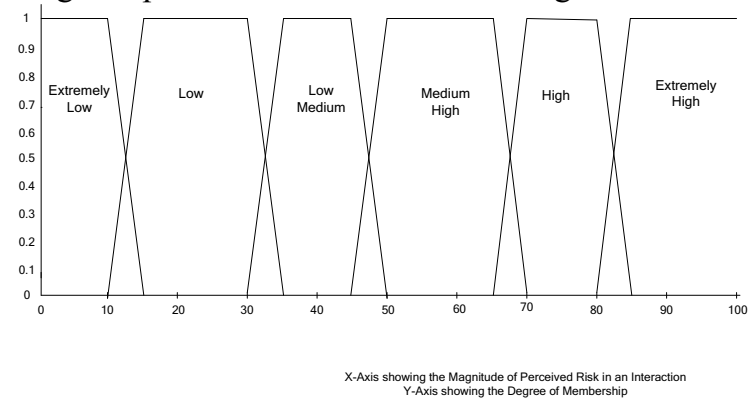

Figure 3: Membership function of the output: Perceived Risk in the Interaction

The output of the fuzzy inference system is determined on the output membership function by the inference engine, which processes the fuzzy sets of the input variables by using the defined rules. In the next section, we define the rules of the fuzzy inference system that help to compute the linguistic level and magnitude of perceived risk in the interaction based on the inputs given to it.

\section{Defining the Rules for the Fuzzy Inference Model}

According to the Mamdani approach, after determining the possibility of the fuzzy sets of each input variable, they must be fed to the inference engine for drawing the conclusion or the output based on the given inputs. The inference engine consists of fuzzy rules by which the conclusions are drawn based on the given inputs. The rules which we use in our model are of the IFTHEN structure. In our fuzzy inference model, there are two inputs based on which the level of perceived risk is determined in the interaction. Each input is further defined by 6 predicates. Hence, the total number of homogenous rules in our system will be: 6 $\mathrm{X} 6=36$. In order to more easily define the rules, we use acronyms for the predicates of our system as defined in Table 1 and define the rules for the fuzzy inference model in Table 2.

\begin{tabular}{|c|c|}
\hline Predicates or the Fuzzy sets & Acronym \\
\hline Extremely Low & 'EL' \\
\hline Low & 'L' \\
\hline Low Medium & 'M' $\mathrm{MH}$ ' \\
\hline Medium High & 'H' \\
\hline High & 'EH' \\
\hline Extremely High &
\end{tabular}

Table 1: Acronyms of the predicates 


\begin{tabular}{|c|c|c|c|c|c|}
\hline & PCF & & LOIP & & PR \\
\hline If & EL & and & EL & then & $\mathrm{L}$ \\
\hline If & $\mathrm{L}$ & and & EL & then & $\mathrm{L}$ \\
\hline If & LM & and & EL & then & LM \\
\hline If & $\mathrm{MH}$ & and & EL & then & $\mathrm{MH}$ \\
\hline If & $\mathrm{H}$ & and & EL & then & $\mathrm{H}$ \\
\hline If & EH & and & EL & then & EH \\
\hline If & EL & and & $\mathrm{L}$ & then & $\mathrm{L}$ \\
\hline If & $\mathrm{L}$ & and & $\mathrm{L}$ & then & LM \\
\hline If & LM & and & $\mathrm{L}$ & then & MH \\
\hline If & $\mathrm{MH}$ & and & $\mathrm{L}$ & then & $\mathrm{H}$ \\
\hline If & $\mathrm{H}$ & and & $\mathrm{L}$ & then & $\mathrm{EH}$ \\
\hline If & $\mathrm{EH}$ & and & $\mathrm{L}$ & then & $\mathrm{EH}$ \\
\hline If & EL & and & LM & then & LM \\
\hline If & $\mathrm{L}$ & and & LM & then & $\mathrm{MH}$ \\
\hline If & LM & and & LM & then & $\mathrm{H}$ \\
\hline If & $\mathrm{MH}$ & and & LM & then & $\mathrm{EH}$ \\
\hline If & $\mathrm{H}$ & and & LM & then & $\mathrm{EH}$ \\
\hline If & EH & and & LM & then & $\mathrm{EH}$ \\
\hline If & EL & and & $\mathrm{MH}$ & then & $\mathrm{MH}$ \\
\hline If & $\mathrm{L}$ & and & $\mathrm{MH}$ & then & $\mathrm{H}$ \\
\hline If & LM & and & $\mathrm{MH}$ & then & $\mathrm{H}$ \\
\hline If & $\mathrm{MH}$ & and & $\mathrm{MH}$ & then & $\mathrm{EH}$ \\
\hline If & $\mathrm{H}$ & and & MH & then & EH \\
\hline If & EH & and & MH & then & EH \\
\hline If & EL & and & $\mathrm{H}$ & then & $\mathrm{H}$ \\
\hline If & $\mathrm{L}$ & and & $\mathrm{H}$ & then & $\mathrm{EH}$ \\
\hline If & LM & and & $\mathrm{H}$ & then & $\mathrm{EH}$ \\
\hline If & MH & and & $\mathrm{H}$ & then & EH \\
\hline If & $\mathrm{H}$ & and & $\mathrm{H}$ & then & EH \\
\hline If & EH & and & $\mathrm{H}$ & then & EH \\
\hline If & EL & and & $\mathrm{EH}$ & then & $\mathrm{EH}$ \\
\hline If & $\mathrm{L}$ & and & $\mathrm{EH}$ & then & $\mathrm{EH}$ \\
\hline If & LM & and & $\mathrm{EH}$ & then & $\mathrm{EH}$ \\
\hline If & $\mathrm{MH}$ & and & $\mathrm{EH}$ & then & $\mathrm{EH}$ \\
\hline If & $\mathrm{H}$ & and & $\mathrm{EH}$ & then & EH \\
\hline If & $\mathrm{EH}$ & and & $\mathrm{EH}$ & then & $\mathrm{EH}$ \\
\hline
\end{tabular}

Table 2: Defining the rules of the fuzzy inference system

The output of the fuzzy inference system will be determined by the degree or strength to which each rule fires. The output of each rule must be aggregated to determine the output fuzzy sets. In our approach, we will use the multiple or additive aggregation operator in order to consider all the consequent parts of the rules which produce an output that relates to the same fuzzy set. The aggregation process gives the output fuzzy sets to which the perceived risk in an interaction corresponds, along with the possibility of them occurring. To determine the scalar output of the fuzzy inference system, the fuzzy sets from the aggregation process must be 'defuzzified'. In our model, we will utilize the centre of gravity or centroid method for defuzzification. The defuzzified scalar output from the centroid method is calculated by:

$$
\mathrm{x}^{\prime}=\frac{\int \mu(x) x d x}{\int \mu(x) d x}
$$

Equation 2 where $\mu(x)$ is the output fuzzy set after the aggregation of the individual implication results.

If the aggregated value from the rules spreads over more than one fuzzy set in the output membership function, then each of those membership functions must be considered while computing the centroid. In the next section, we will demonstrate the process for determining the linguistic level of perceived risk in the interaction by using the proposed fuzzy inference model.

\section{Determining the Linguistic levels of Perceived Risk in an Interaction}

To explain the proposed approach of determining the linguistic level of perceived risk, let us consider an interaction scenario where the risk assessing agent ' $\mathrm{A}$ ' determines the focal elements and their possibility of occurrence from the UoD of the input variables as shown in Figure 4 and Figure 5.

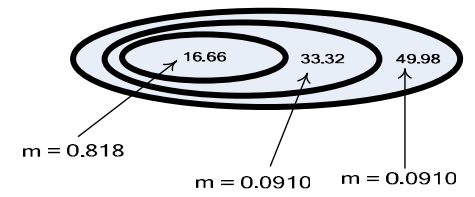

Figure 4: The focal elements and their degree of evidence for the input: Possible Consequences of Failure

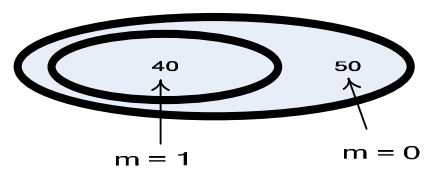

Figure 5: The focal elements and their degree of evidence for the input: Loss of Investment Probability in an Interaction

Once the focal elements and their degree of evidence for the input constituents PCF and the LOIP have been determined, the risk assessing agent by using equation 1 should transform the focal elements of each input variable to its defined fuzzy sets. Determining the fuzzy sets for the input variable PCF:

$\Pi_{\mathrm{PCF}}(\mathrm{EL})=\max \left\{\min \left[\pi(\mathrm{x}), \mathrm{DOM}_{\mathrm{EL}}(\mathrm{x})\right]\right\}=0$

$\Pi_{\mathrm{PCF}}(\mathrm{L})=1$

$\Pi_{\mathrm{PCF}}(\mathrm{LM})=0.182$

$\Pi_{\mathrm{PCF}}(\mathrm{MH})=0.0910$

$\Pi_{\mathrm{PCF}}(\mathrm{H})=0$

$\Pi_{\mathrm{PCF}}(\mathrm{EH})=0$ 
Similarly, the fuzzy sets of the input variable LOIP are:

$$
\begin{aligned}
& \Pi_{\text {LOIP }}(\mathrm{EL})=0 \\
& \Pi_{\text {LOIP }}(\mathrm{L})=0 \\
& \Pi_{\text {LOIP }}(\mathrm{LM})=1 \\
& \Pi_{\text {LOIP }}(\mathrm{MH})=0 \\
& \Pi_{\text {LOIP }}(\mathrm{H})=0 \\
& \Pi_{\text {LOIP }}(\mathrm{EH})=0
\end{aligned}
$$

Once the fuzzy sets, along with the possibility of occurrence of each predicate from the membership function of the input variables, have been determined, the risk assessing agent should then evaluate and aggregate the rules shown in Table 2 to determine the output fuzzy sets on the output membership function. The fuzzy sets of the output variable PR determined from the evaluation of the rules are:

$$
\begin{aligned}
& \Pi_{\mathrm{PR}}(\mathrm{EL})=0 \\
& \Pi_{\mathrm{PR}}(\mathrm{L})=0 \\
& \Pi_{\mathrm{PR}}(\mathrm{LM})=0 \\
& \Pi_{\mathrm{PR}}(\mathrm{MH})=1 \\
& \Pi_{\mathrm{PR}}(\mathrm{H})=0.182 \\
& \Pi_{\mathrm{PR}}(\mathrm{EH})=0.0910
\end{aligned}
$$

The determined linguistic level of perceived risk can be represented as a possibility distribution as shown in Figure 6 . The centroid of the shaded area determined by using equation 2 is 61.72 .

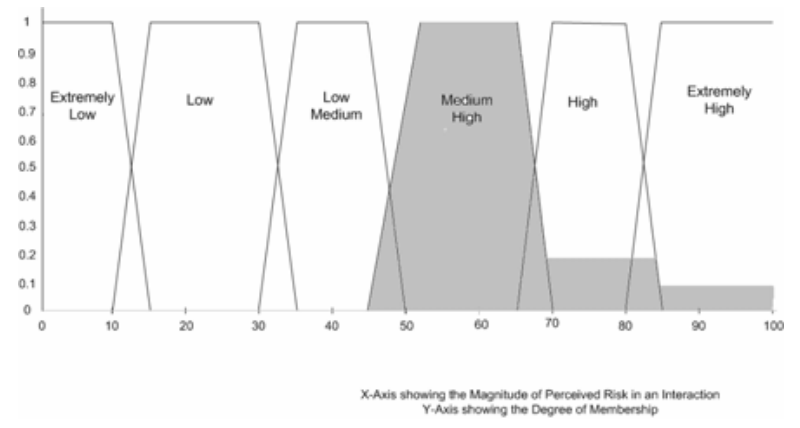

Figure 6: The linguistic level of Perceived Risk in the interaction

The risk assessing agent ' $A$ ' can utilize the determined semantic levels of perceived risk in making an informed decision of forming an interaction with the risk assessed agent. As the determined linguistic level of perceived risk has semantics associated with it, the risk assessing agent can utilize them better, while determining their impact on its risk propensity or risk taking attitude to make an informed decision of its future course in the interaction with the risk assessed agent. This is our future work, where the risk assessing agent carries out the steps of risk management to make an informed decision of its future course of interaction with the risk assessed agent, based on the linguistic level of perceived risk determined in forming an interaction with it.

\section{Conclusion}

In this paper, we proposed a methodology by which the risk assessing agent can determine the linguistic level of perceived risk in forming an interaction with a risk assessed agent. We utilize a fuzzy inference system to achieve this. By determining the different levels and magnitude of perceived risk, the risk assessing agent can get an idea of how its interaction with the risk assessed agent will proceed, if it chooses that agent to interact with. It can also utilize each level of perceived risk and its magnitude of occurrence according to its risk propensity nature to make an informed interaction-based decision with that agent. This is our future work.

\section{References}

[1] R.C. Mayer, J.H. Davis, and F. D. Schoorman, "An Integrative Model of Organizational Trust," The Academy of Management Review, vol. 20, pp. 709734, 1995.

[2] Omar Khadeer Hussain, Elizabeth Chang, Farookh Khadeer Hussain, and Tharam S. Dillon, "A methodology to quantify failure for risk-based decision support system in digital business ecosystems," Data \& Knowledge Engineering, vol. 63, pp. 597-621, 2007.

[3] Omar K. Hussain, Elizabeth Chang, Farookh K. Hussain, and Tharam S. Dillon, "Towards Quantifying the Possible Risk in e-commerce Interactions for RDSS," in Proceedings of the IEEE International Conference on e-Business Engineering (ICEBE'07), Hong Kong, China 2007, pp. 89-96.

[4] Omar Hussain, Elizabeth Chang, Tharam Dillon, and F. Hussain, "Determining the Level of Perceived Risk in e-business Web 2.0 Interactions," Accepted for Publication at 2008 IEEE International Conference on e-Business Engineering (ICEBE'08,) Xian, China, 2008.

[5] Riza C. Berkan and S. L. Trubatch, Fuzzy Systems Design Priciples. New York: IEEE Press, 1997. 